

\title{
APPLICATION OF A REYNOLDS STRESS TURBULENCE MODEL TO THE COMPRESSIBLE SHEAR LAYER
}

\author{
S. Sarkar ${ }^{1}$ \\ Institute for Computer Applications in Science and Engineering \\ NASA Langley Research Center \\ Hampton, VA 23665 \\ and \\ L. Balakrishnan \\ Old Dominion University \\ Norfolk, VA 23508
}

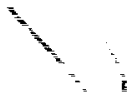

\section{ABSTRACT}

Theoretically based turbulence models have had success in predicting many features of incompressible, free shear layers. However, attempts to extend these models to the highspeed, compressible shear layer have been less effective. In the present work, the compressible shear layer was studied with a second-order turbulence closure, which initially used only variable density extensions of incompressible models for the Reynolds stress transport equation and the dissipation rate transport equation. The quasi-incompressible closure was unsuccessful; the predicted effect of the convective Mach number on the shear layer growth rate was significantly smaller than that observed in experiments. Having thus confirmed that compressibility effects have to be explicitly considered, a new model for the compressible dissipation was introduced into the closure. This model is based on a low Mach number, asymptotic analysis of the Navier-Stokes equations, and on direct numerical simulations of compressible, isotropic turbulence. The use of the new model for the compressible dissipation led to good agreement of the computed growth rates with the experimental data. Both the computations and the experiments indicate a dramatic reduction in the growth rate when the convective Mach number is increased. Experimental data on the normalized maximum turbulence intensities and shear stress also show a reduction with increasing Mach number. The computed values are in accord with this trend.

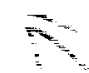

\footnotetext{
${ }^{1}$ This research was supported by the National Aeronautics and Space Administration under NASA Contract No. NAS1-18605 while the author was in residence at the Institute for Computer Applications in Science and Engineering (ICASE), NASA Langley Center, Hampton, VA 23665.
} 
$\equiv$

$=$

$\equiv$

$\bar{\equiv}$

$\overline{\underline{\underline{\underline{E}}}}$
$\equiv$
$\equiv$
-

$\underline{\underline{\underline{\underline{ }}}}$ 


\section{Introduction}

The reduced growth rate of the high-speed, compressible shear layer relative to its low-speed counterpart has been confirmed in several experimental studies, for example, in the recent investigations of Papamoschou and Roshko', and Elliott and Samimy ${ }^{2}$. However, variable density extensions of incompressible turbulence models, without any explicit compressibility terms, have failed to predict the significant decrease in the spreading rate caused by an increase in the convective Mach number. This has led to attempts by $\mathrm{Oh}^{3}$, Vandromme, and Dussauge and Quine ${ }^{5}$, among others, to make phenomenological modifications to incompressible turbulence models, in order to obtain successful predictions of the compressible mixing layer. Recently, Sarkar et al. ${ }^{6}$ and $Z_{e m a n}{ }^{7}$ have recognized the importance of an additional contribution to the turbulent dissipation rate, which is generated by the non-negligible fluctuating dilatation in compressible turbulence. The additional term - the compressible dissipation - has been modeled by Sarkar et al. ${ }^{6}$; this model is based on a low Mach number, asymptotic analysis of the compressible Navier-Stokes equations and is calibrated with reference to direct numerical simulations of compressible, isotropic turbulence. The present paper applies the model of the compressible dissipation to the high-speed shear layer within the framework of a second-order turbulence closure. A schematic of the shear layer is given in Fig. 1.

The paper is organized in the following manner. In Section 2 the exact governing equations are given, and the turbulence models constituting the second-order closure are described. The numerical procedure is outlined in Section 3. The results of the calculations with the second-order closure are given in Section 4 , and conclusions are presented in Section 5 .

\section{The governing equations}

We obtain the equations for the mean variables by first decomposing each variable into a mean component and a fluctuating component, and then averaging the equations for the 
following variables: the density $\rho$, the velocity $u_{i}$ and the total energy $E$. The total energy $\mathrm{E}$ is defined by

$$
E=\frac{u_{i} u_{i}}{2}+C_{v} T
$$

where $T$ denotes the static temperature, and $C_{v}$ is the specific heat at constant volume. The Reynolds decomposition of an instantaneous variable $\phi$ into its mean and fluctuating components is

$$
\phi=\bar{\phi}+\phi^{\prime \prime}
$$

where, by definition, $\overline{\phi^{\prime \prime}}=0$. The Favre decomposition of an instantaneous variable is also used in compressible turbulence, primarily because the resulting structure of the averaged inertial terms is simpler; this decomposition is given by

$$
\phi=\tilde{\phi}+\phi^{\prime}
$$

where $\tilde{\phi}$ is the density-weighted Reynolds a verage,

$$
\tilde{\phi}=\frac{\overline{\rho \phi}}{\bar{\rho}}
$$

The overbar over a variable is used to denote a conventional Reynolds average, while the overtilde is used to denote the Favre average. A single superscript ' represents fluctuations with respect to the Favre average, while a double superscript " signifies fluctuations with respect to the Reynolds average. The conventional Reynolds average of Favre fluctuations is non-zero, in particular, $\overline{\phi^{\prime}}=-\overline{\rho^{\prime \prime} \phi^{\prime \prime}} / \bar{\rho}$. After averaging the instantaneous Navier-Stokes equations, the following mean equations are obtained:

Conservation of mass:

$$
\partial_{t}(\bar{\rho})+\left(\bar{\rho} \tilde{u}_{k}\right)_{, k}=0
$$

Conservation of momentum:

$$
\partial_{t}\left(\bar{\rho} \tilde{u}_{i}\right)+\left(\bar{\rho} \tilde{u}_{k} \tilde{u}_{i}\right)_{, k}=-\bar{p}_{, \mathbf{i}}+\bar{\tau}_{i k, k}-\left(\bar{\rho} \widetilde{u_{i}^{\prime} u_{k}^{\prime}}\right)_{, k}
$$




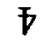

(6)

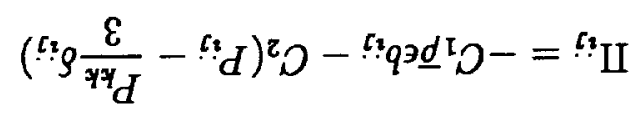

'uoţ

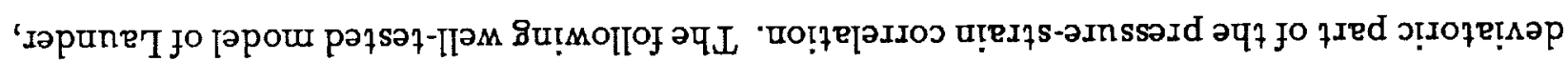

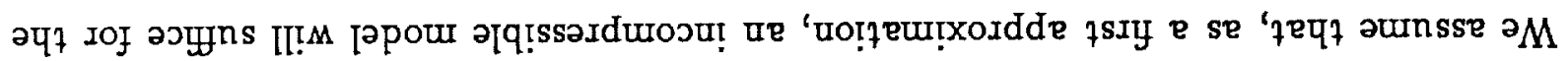
'uo!fenba quodsuejq ssazqs-spjouKay

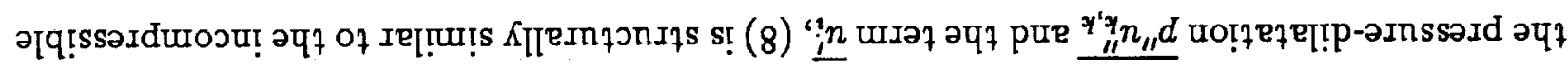

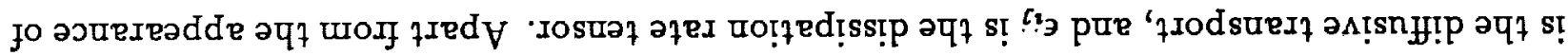

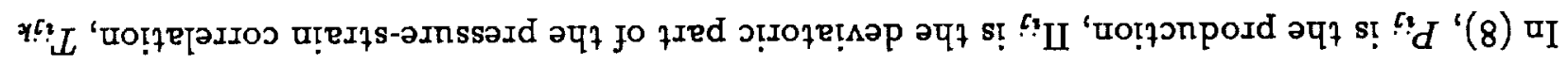

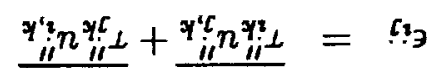

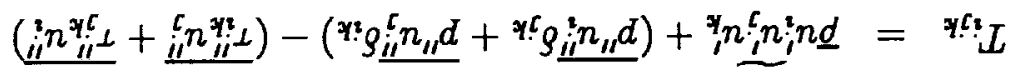

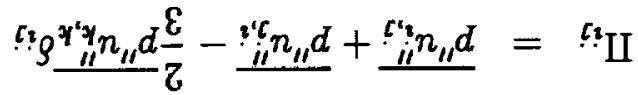

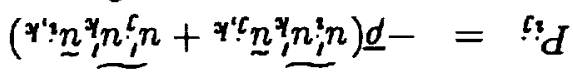

әЈәчМ

(8)

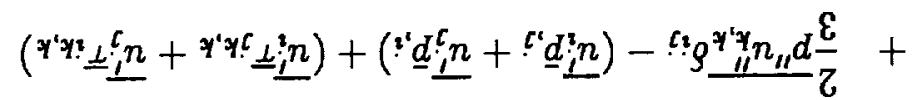

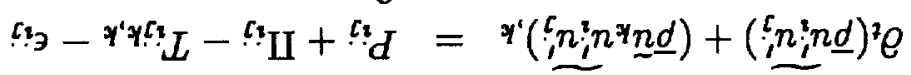

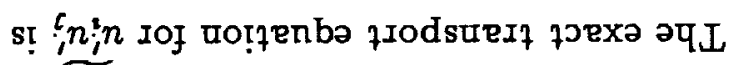

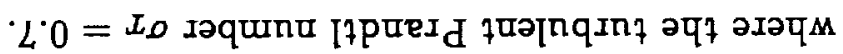

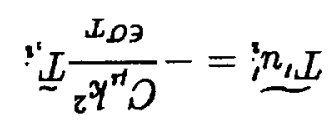

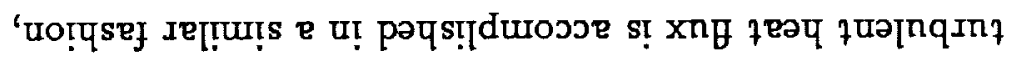

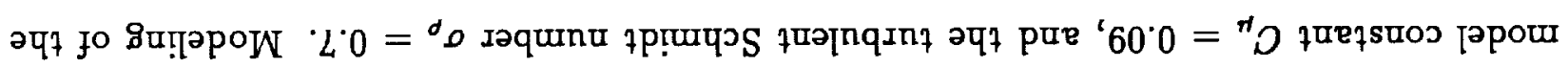

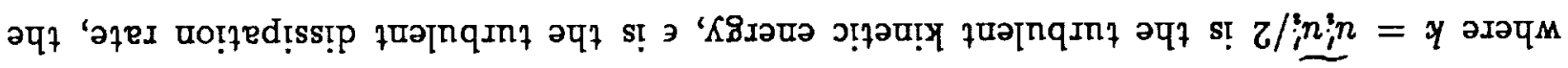

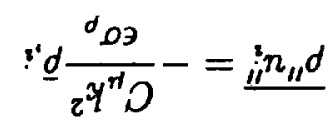

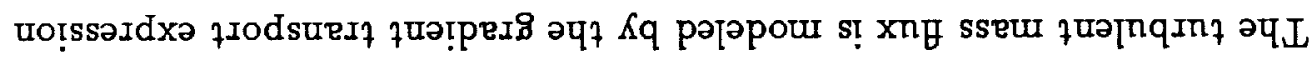




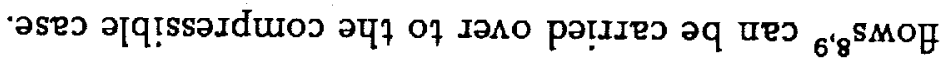

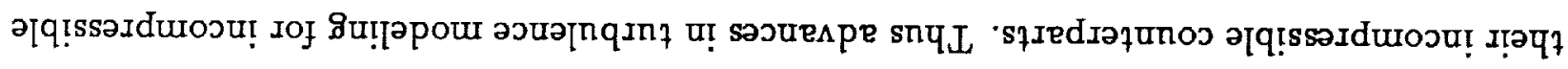

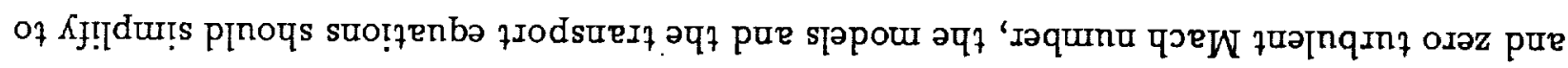

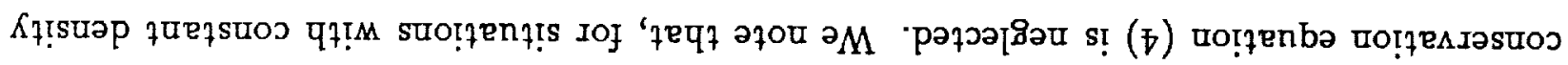

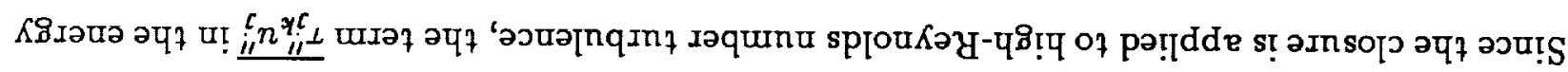

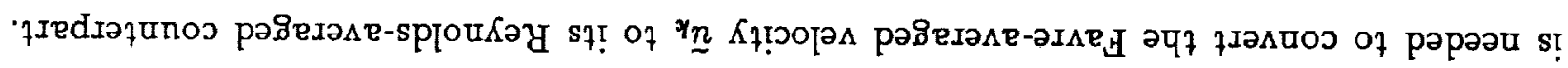

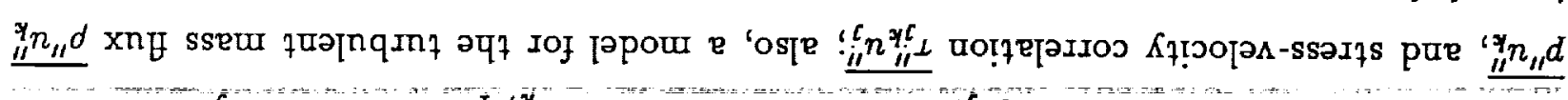

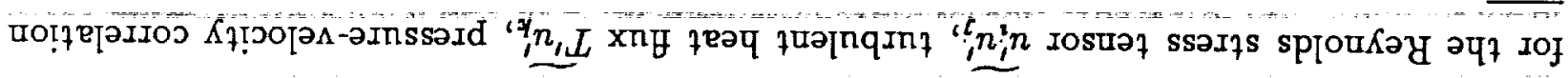

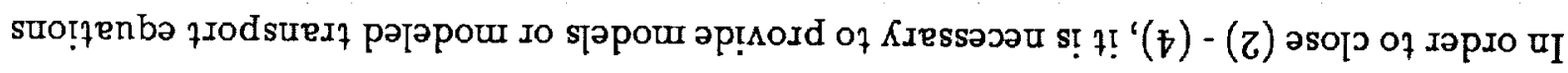

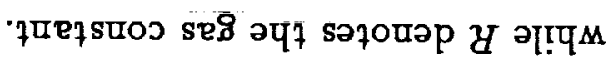

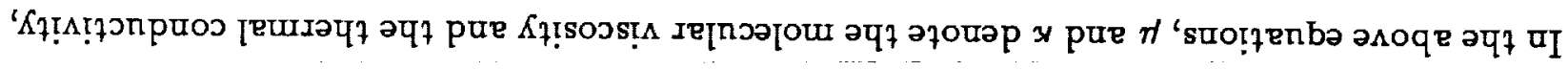

$$
\underline{L} y \underline{d}=\underline{\bar{d}}
$$

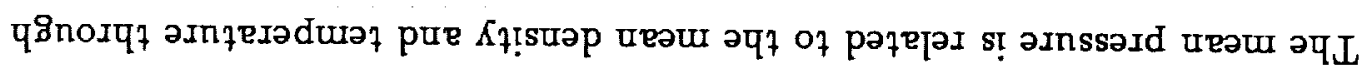

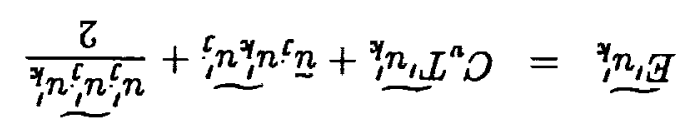

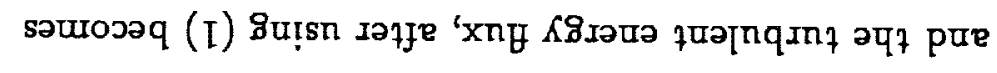

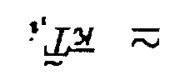

$$
\begin{aligned}
& \underline{\mathfrak{i}^{\prime}} \underline{L^{x}}=\underline{?} \underline{\underline{b}}
\end{aligned}
$$

s! $x n_{f}$ ұеәq पеәш әчұ әләपм

$(\mp)$

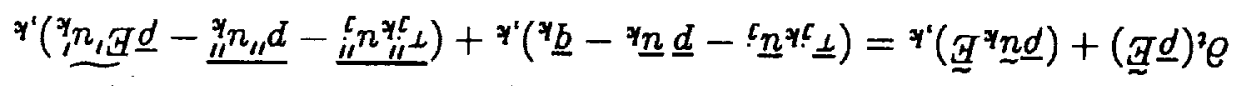

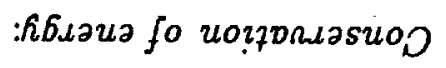

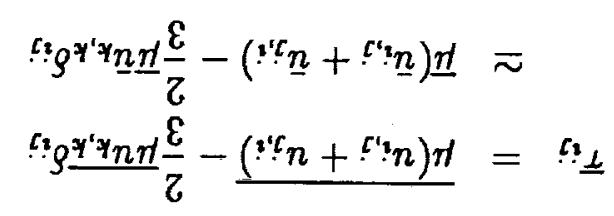

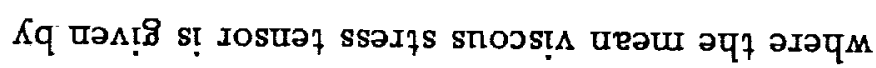


where the anisotropy tensor $b_{i j}$ is given by

$$
b_{i j}=\frac{\widetilde{u_{i}^{T} u_{j}^{\prime}}}{q^{2}}-\frac{\delta_{i j}}{3}
$$

and $q^{2}=\widetilde{u_{m}^{\prime} u_{m}^{\prime}}=2 k$ denotes the trace of the Reynolds stress tensor. In (9) the model constants are

$$
C_{1}=3.0 \quad, \quad C_{2}=0.6
$$

Since the primary aim of the paper is to study the influence of terms that arise solely from flow compressibility, we do not use more sophisticated incompressible pressure-strain models, such as those proposed by Shih and Lumley ${ }^{11}$; Fu, Launder and Tselepidakis ${ }^{12}$; and Speziale, Sarkar and Gatski ${ }^{13}$.

The dissipation rate tensor $\epsilon_{i j}$ is commonly believed to be isotropic at high turbulence Reynolds numbers, leading to the model

$$
\epsilon_{i j}=\frac{2}{3} \bar{\rho} \epsilon \delta_{i j}
$$

where the turbulent dissipation rate $\epsilon$ is given by

$$
\bar{\rho} \epsilon=\overline{\tau_{k l}^{\prime \prime} u_{k, l}^{\prime \prime}}
$$

The viscous stress in a compressible fluid is

$$
\tau_{i j}=\mu\left(u_{i, j}+u_{j, i}\right)-\frac{2}{3} \mu u_{k, k} \delta_{i j}
$$

where we have neglected the bulk viscosity. As shown in Sarkar et al. ${ }^{6}$, substitution of (12) into (11), followed by some algebraic manipulation, gives

$$
\bar{\rho} \epsilon=\bar{\rho}\left(\epsilon_{s}+\epsilon_{c}\right)
$$

where

$$
\epsilon_{a}=\bar{\nu} \overline{\omega_{i}^{\prime \prime} \omega_{i}^{\prime \prime}}
$$

and

$$
\epsilon_{c}=\frac{4}{3} \bar{\nu} \overline{d^{\prime \prime 2}}
$$


Here $\omega_{i}^{\prime \prime}$ is the fluctuating vorticity, and $d^{\prime \prime}=u_{k, k}^{\prime \prime}$ is the fluctuating dilatation. The decomposition (13) of the turbulent dissipation rate $\epsilon$ into the solenoidal dissipation $\epsilon_{s}$ and the compressible dissipation $\epsilon_{\mathrm{c}}$ is asymptotically valid for high-Reynolds number turbulence, and is exact for constant-viscosity homogeneous turbulence. Because of the explicit compressible contribution to the turbulent dissipation rate, the treatment of $\epsilon$ has to be modified with respect to the incompressible case. Developing an appropriate, direct modification of the transport equation for $\epsilon$ is a difficult proposition, because the exact transport equation for $\epsilon$ is complicated for the incompressible case, and even more so for the compressible case. Also, as discussed by Speziale ${ }^{14}$, the addition of new terms into the $\epsilon$ transport equation has often led to unintended, deleterious effects in homogeneous flow. In the present work, we adopt a simpler alternative. The incompressible form of the dissipation equation is retained as a transport equation for $\epsilon_{s}$; such an approach is valid, because $\epsilon_{s}$ is not affected by moderate levels of compressibility ${ }^{6}$. It remains to model $\epsilon_{c}$; we choose the simple, algebraic model of Sarkar et al. ${ }^{6}$,

$$
\epsilon_{c}=\alpha_{1} \epsilon_{s} M_{t}^{2}
$$

which is motivated by an asymptotic analysis of the compressible Navier-Stokes equations with $M_{t}$ as the small parameter. Here $M_{t}$ denotes the turbulent Mach number defined by $M_{t}=\sqrt{q^{2} / \gamma R \tilde{T}}$, and $\tilde{T}$ is the Favre-averaged temperature. Finally, the model for $\epsilon_{i j}$ becomes

$$
\epsilon_{i j}=\frac{2}{3} \bar{\rho} \epsilon_{s}\left(1+\alpha_{1} M_{t}^{2}\right) \delta_{i j}
$$

The model constant was set as $\alpha_{1}=1$ with reference to direct numerical simulations of the decay of isotropic, compressible turbulence. Zeman ${ }^{7}$ has also used a similar decomposition of the turbulent dissipation rate, and after assuming that eddy shocklets occur in high-speed flows, he derives a model for the contribution of these eddy shocklets to the compressible dissipation.

In the present work, we assume that the bulk viscosity $\mu_{v}=0$. If the bulk viscosity $\mu_{v}$ is non-negligible, for example in polyatomic gases, there is an additional turbulent dissipation 
term $\bar{\rho} \epsilon_{b}=\overline{\mu_{v}} \overline{d^{\prime \prime 2}}$ which can be modeled as $\epsilon_{b}=\alpha_{2} \epsilon_{s} M_{t}^{2}$. If the value of $\mu_{v}$ is known, $\alpha_{2}$ can be easily determined from $\alpha_{1}$ by the relation $\alpha_{2}=3 \overline{\mu_{v}} \alpha_{1} / 4 \bar{\mu}$.

The pressure-dilatation $\overline{p^{\prime \prime} d^{\prime \prime}}$, which is not necessarily single-signed (i.e; it is neither positive semi-definite or negative semi-definite) like the compressible dissipation, is a more difficult term to model. Low Mach number asymptotic theory ${ }^{15,6}$ suggests that $\overline{p^{\prime \prime} d^{\prime \prime}}$ is negligible compared to $\epsilon_{c}$, and from direct simulations ${ }^{6}$ it appears that in isotropic, moderate Mach number turbulence $\overline{p^{\prime \prime} d^{\prime \prime}}$ is appreciably smaller than $\epsilon_{c}$. In the present closure, we will neglect $\overline{p^{\prime \prime} d^{\prime \prime}}$ relative to $\epsilon_{\mathrm{c}}$.

The diffusive transport $T_{i j k}$ is modeled by a gradient transport expression,

$$
T_{i j k}=-C_{s} \bar{\rho} \frac{\left(q^{2}\right)^{2}}{\epsilon}\left[\left(\widetilde{u_{i}^{\prime} u_{j}^{\prime}}\right)_{, k}+\left(\widetilde{u_{j}^{\prime} u_{k}^{\prime}}\right)_{, i}+\left(\widetilde{u_{i}^{\prime} u_{k}^{\prime}}\right)_{, j}\right]
$$

where $C_{s}=0.018$. The quantity $\overline{u_{i}^{\prime}}$ is related to the turbulent mass flux $\overline{\rho^{\prime \prime} u_{i}^{\prime \prime}}$ by

$$
\overline{u_{i}^{\prime}}=-\frac{\overline{\rho^{\prime \prime} u_{i}^{\prime \prime}}}{\bar{\rho}}
$$

and after using (6) for the mass flux, we obtain the model

$$
\overline{u_{i}^{\prime}} \simeq \frac{C_{\mu} k^{2}}{\bar{\rho} \epsilon \sigma_{\rho}} \bar{\rho}_{, i}
$$

The standard high-Reynolds number form of the dissipation rate equation is used as the transport equation for $\epsilon_{s}$,

$$
\partial_{t}\left(\bar{\rho} \epsilon_{s}\right)+\left(\bar{\rho} \tilde{u}_{k} \epsilon_{s}\right)_{, k}=-C_{\epsilon 1} \frac{\epsilon_{s}}{k} \bar{\rho} \widetilde{u_{i}^{\prime} u_{j}^{\prime} \tilde{u}_{i, j}}-C_{\epsilon 2} \bar{\rho} \frac{\epsilon_{s}^{2}}{k}+\left(C_{\epsilon} \frac{\bar{\rho} k}{\epsilon_{s}} \widetilde{u_{k}^{\prime} u_{l}^{\prime} \epsilon_{s, l}}\right)_{, k}
$$

The model coefficients in (21) are

$$
C_{\epsilon 1}=1.44 \quad, \quad C_{\epsilon 2}=1.90 \quad, \quad C_{\epsilon}=0.15
$$

For the present problem, we need to solve (2)-(4), along with the equation of state, to obtain the mean variables: $\bar{\rho}, \tilde{U}, \tilde{V}$, and $\tilde{E}$. In the case of the plane shear layer, the Reynolds stress tensor has four non-zero components: $\widetilde{u^{\prime} v^{\prime}}, \widetilde{u^{\prime 2}}, \widetilde{v^{\prime 2}}$ and $\widetilde{w^{\prime 2}}$, which are solved by the corresponding components of (8). The equation for the solenoidal dissipation rate $\epsilon_{s}$ completes the set of governing equations. Thus a system of nine coupled, non-linear, partial differential equations along with an appropriate set of initial and boundary conditions must be solved. 


\section{Method of Solution of the Governing Equations}

The transport equations for the mean flow and Reynolds stresses are written in the physical domain and must be transformed to the computational domain using an appropriate coordinate transformation. For the physical problem under consideration, an algebraic grid generation technique is used to generate the mesh. In the physical domain a uniform grid is used in the axial direction and in the normal direction the grid lines are clustered near regions where strong gradients exist. A uniform mesh is used in the computational domain. The governing equations are first cast into a vector form, where $U$ is the dependent variable vector consisting of nine components, the vectors $F$ and $G$ respectively denote the $x$ and $y$ flux vectors, and $H$ is the source vector containing the terms causing production, destruction and redistribution of the Reynolds stresses. To numerically obtain the solution for the vector $U$, the governing equations are then transformed from the physical domain to the computational domain, giving the following system of equations,

$$
\frac{\partial \hat{U}}{\partial t}+\frac{\partial \hat{F}}{\partial \xi}+\frac{\partial \hat{G}}{\partial \eta}=\hat{H}
$$

where

$$
\begin{aligned}
\hat{U}=J U \quad & \quad \hat{H}=J H \\
\hat{F}=F y_{\eta}-G x_{\eta} & , \quad \hat{G}=G x_{\xi}-F y_{\xi} \quad, \quad J=x_{\xi} y_{\eta}-y_{\xi} x_{\eta} .
\end{aligned}
$$

In (23), a superscript () denotes quantities in the transformed system, $\left(x_{\xi}, x_{\eta}, y_{\xi}, y_{\eta}\right)$ represent the metrics of the transformation, and $J$ denotes the Jacobian of the transformation. If the physical grid is given, the metrics and the Jacobian of the transformation can be easily computed.

The governing equations are integrated explicitly in time using the unsplit MacCormack predictor-corrector scheme. During a specific numerical sweep, the inviscid fluxes and the first-derivative terms in the source vector $\mathrm{H}$ are backward differenced in the predictor step and forward differenced in the corrector step. Second-order central differences are used for 
the viscous and heat flux terms. Hence the complete scheme for both the predictor and corrector steps can be expressed as follows

Predictor:

$$
\begin{aligned}
\Delta \hat{U}_{i, j}{ }^{\overline{n+1}} & =-\Delta t\left(\frac{\nabla \xi \hat{F}_{i, j}{ }^{n}}{\Delta \xi}+\frac{\nabla_{\eta} \hat{G}_{i, j}{ }^{n}}{\Delta \eta}-\hat{H}_{i, j}{ }^{n}\right) \\
\hat{U}_{i, j}{ }^{\overline{n+1}} & =\hat{U}_{i, j}{ }^{n}+\Delta \hat{U}_{i, j}{ }^{\overline{n+1}}
\end{aligned}
$$

Corrector:

$$
\begin{aligned}
& \Delta \hat{U}_{i, j}{ }^{n+1}=-\Delta t\left(\frac{\triangle_{\xi} \hat{F}_{i, j}^{\overline{n+1}}}{\Delta \xi}+\frac{\triangle_{\eta}{\hat{G_{i, j}}}^{\overline{n+1}}}{\Delta \eta}-{\hat{H_{i, j}}}^{\overline{n+1}}\right) \\
& {\hat{U_{i, j}}}^{n+1}=\frac{1}{2}\left({\hat{U_{i, j}}}^{n}+{\hat{U_{i, j}}}^{\overline{n+1}}+\Delta \hat{U}_{i, j}{ }^{n+1}\right)
\end{aligned}
$$

The composite numerical scheme is second-order accurate in both time and space and, being an explicit scheme, is conditionally restricted by the Courant and viscous stability limits of the governing equations. The solution procedure requires no scalar or block tridiagonal inversions. The flow field is advanced from time level $n$ to $n+1$ and this process is continued until the desired integration time or steady state has been reached. Since the Reynolds stress transport equations contain stiff source terms, the maximum CFL number used in the computation was limited to 0.5 .

The numerical code used in this study is a two-dimensional, elliptic, Navier-Stokes solver (SPARK2D ${ }^{16}$ ) written in a generalized body-oriented coordinate system. As such, various two-dimensional free shear flows and wall bounded flows can be handled by the numerical code. The code in its original form used a second-order spatially and temporally accurate, two-step MacCormack scheme. The latter versions of the code employ a variety of higherorder compact algorithms ${ }^{17}$ (4th and 6 th order) and various upwind schemes. Local time stepping and residual smoothing options are also available in the code to accelerate the convergence to steady state. Both laminar reacting and non-reacting flows can be easily handled by the code. In the present research work, the capabilities of SPARK2D are further enhanced by adding a second-order Reynolds stress model as a turbulence closure. 
Since the governing equations are elliptic in nature, the boundary conditions have to be specified along all four boundaries. These include inflow, outflow and outer boundaries (lower and upper boundaries) respectively. At the inflow boundary $(x=0.0)$, profiles are specified for the velocities, static pressure, static temperature, turbulent stresses and the turbulent dissipation rate. Since we are interested in the downstream fully-developed regime, the specific form of the inlet profiles is not crucial.

The outer boundaries always remain in the free-stream and the appropriate boundary condition is to assume that the normal derivative of the flow variables vanish along those boundaries. The gradient boundary conditions, not only preserve the free-stream values along the outer boundaries but also provide nonreflective conditions for the outgoing waves. The outflow boundary $\left(x=x_{\max }\right)$ is always supersonic, and hence the values of mean flow and turbulence quantities are determined by zeroth-order extrapolation from upstream values. Along with the boundary conditions, the governing equations also require a set of initial conditions. The initial conditions at time $t=0$ for all the variables are obtained by simply propagating the inflow profiles throughout the computational domain. Having specified all the boundary and initial data the equations are marched in time until the residual based on $\bar{\rho} \tilde{U}$ decreases by six orders of magnitude, indicating that a converged solution has been obtained.

\section{Results}

It is known that the fully-developed, high-Reynolds number shear layer spreads linearly, and that the growth rate $d \delta / d x$ satisfies the relation.

$$
\frac{d \delta}{d x}=C_{\delta}\left(\frac{U_{1}-U_{2}}{U_{1}+U_{2}}\right)
$$

where $\delta(x)$ denotes the width of the shear layer, and $C_{\delta}$ is approximately constant. The shear layer thickness $\delta(x)$ has been defined in several ways by previous investigators; in the present work, $\delta(x)$ represents the distance between the two cross-stream positions where the normalized streamwise velocity $U^{*}=\left(U-U_{2}\right) /\left(U_{1}-U_{2}\right)$ is respectively 0.1 and 0.9 . The 
fully-developed nature of the shear layer is also characterized by the maximum values of the normalized turbulent stresses $\sigma_{u}, \sigma_{v}, \sigma_{w}$ and $\sigma_{u v}$ reaching constants; where

$$
\begin{aligned}
\sigma_{u} & =\sqrt{\widetilde{u^{\prime 2}}} /\left(U_{1}-U_{2}\right) \\
\sigma_{v} & =\sqrt{\widetilde{v^{\prime 2}}} /\left(U_{1}-U_{2}\right) \\
\sigma_{w} & =\sqrt{\widetilde{w^{\prime^{2}}}} /\left(U_{1}-U_{2}\right) \\
\sigma_{u v} & =\sqrt{-\widetilde{u^{\prime} v^{\prime}}} /\left(U_{1}-U_{2}\right)
\end{aligned}
$$

Figs. 2-6 show results for a particular set of conditions for the shear layer between two streams of air. The high-speed stream had a velocity $U_{1}=2500 \mathrm{~m} / \mathrm{s}$ while the low-speed stream had a velocity $U_{2}=800 \mathrm{~m} / \mathrm{s}$. The thermodynamic quantities in the two incident streams were equal and were prescribed as $T_{1}=800 \mathrm{~K}, p_{1}=1 \mathrm{~atm}$, and $\rho_{1}=0.44 \mathrm{~kg} / \mathrm{m}^{3}$. When the ratio of specific heats $\gamma$ has the same value in the two streams, the convective Mach number $M_{c}$ is given $\mathrm{by}^{1}$,

$$
M_{c}=\frac{U_{1}-U_{2}}{a_{1}+a_{2}}
$$

where $a_{1}$ and $a_{2}$ are the respective speeds of sound in the two layers. The case described by Figs. 2-6 corresponds to $M_{c}=1.5$. The computational domain for this case was a rectangle of dimensions $0.1 \mathrm{~m} \times 0.05 \mathrm{~m}$ with a $201 \times 51$ grid overlaying it. The grid spacing was uniform in the streamwise direction and stretched in the cross-stream direction. Based on comparison with results using other grid spacings, the resolution of the $201 \times 51$ grid for the computational domain was found to be sufficient to provide practically grid-independent results for the mean velocity and turbulent stress profiles. As an example of the grid sensitivity of the calculated solution, increasing the number of grid points by a factor of approximately 1.7 changed the values of $C_{\delta}$, and the maximum values of $\sigma_{u}, \sigma_{v}, \sigma_{w}$ and $\sigma_{u v}$ by less than $2 \%$ from the values corresponding to the $201 \times 51$ grid.

Fig. 2 shows that the shear layer thickness $\delta(x)$ increases linearly after an initial development phase. In Fig. 3 the normalized streamwise mean velocity $U^{*}$ at the inlet, outlet and two intermediate locations is plotted as a function of the similarity variable $\eta=\left(y-y_{c}\right) / \delta$, where $y$ is the local cross-stream coordinate and $y_{c}$ is the cross-stream coordinate where 
$U^{*}=0.5$. It is evident from Figs. 2 and 3 that, at the outflow boundary of the computational box, the linearly growing regime is well-established and the mean velocity has reached its self-similar profile. The similarity mean velocity profile of Fig. 3 is somewhat asymmetric with respect to its center $\eta=0$ and indicates a greater penetration into the low-speed side than the corresponding penetration into the upper, high-speed side of the domain. Fig. 4 shows the mean temperature profile across the shear layer. There is a sharp increase of the temperature in the core of the shear layer due to the large velocity gradients there. Figs. 5 and 6 show profiles of the normalized streamwise turbulence intensity $\sigma_{u}$ and the normalized shear stress $\sigma_{u v}$. All the components of the normalized Reynolds stress tensor reach their self-similar profiles at the exit of the computational box.

The growth rate parameter $C_{\delta}$ and the maximum values of the normalized Reynolds stresses $\sigma_{u}, \sigma_{v}, \sigma_{w}$ and $\sigma_{u v}$ are nominally constant for the incompressible shear layer. However, it is clear from the experimental data of Figs. 7 and 8 that these quantities show a systematic decrease when the convective Mach number $M_{c}$ increases. In Fig. 7 , the incompressible value $\left(C_{\delta}\right)_{0}$, which was obtained by calculating a case with a small $M_{c}$, was used to normalize the growth rate parameter $C_{\delta}$. Fig. 7 indicates that the Reynolds stress calculations without the compressibility model (16) show only a modest decrease in the growth rate parameter. However, introduction of the model for the compressible dissipation leads to good agreement with both the experimentally observed trends of the sharp decrease in the growth rate, and the later flattening of the growth rate curve in the high Mach number range. It is evident from Fig. 8 that computations with the compressible dissipation model are in qualitative agreement with the observed trend of a decrease in the maximum normalized Reynolds stress components with an increase in $M_{c}$.

Growth rate curves for various values of $\alpha_{1}$ are shown in conjunction with the Langley experimental curve ${ }^{18}$ in Fig. 9. Increasing $\alpha_{1}$ from its recommended value of 1.0 leads to a sharper reduction of the growth rate before the eventual flattening out at high convective Mach numbers. The flattening of the growth rate curve for high $M_{c}$ is due to the maximum 
turbulent Mach number $M_{t}$ asymptoting to an equilibrium level (as shown in Fig. 10), and consequent leveling out of the compressible contribution to the turbulent dissipation rate.

The model of Sarkar et al. ${ }^{6}$ for the compressible dissipation, which was used in the present work, has also been applied by Wilcox ${ }^{19}$ to some supersonic and hypersonic flows within the framework of a $k-\omega$ turbulence closure. Wilcox's study concludes that the addition of this model of the compressible dissipation leads to the experimentally observed reduction in the growth rate of the compressible shear layer, leads to values of skin friction in adiabatic boundary layers that are somewhat lower than the measured values, and results in an improved prediction of the separation bubble size in a shock-boundary layer interaction problem.

\section{Conclusions}

Initially, a second-order turbulence closure without any explicit compressibility models was applied to the high-speed shear layer. The results confirmed earlier conclusions ${ }^{18,20,21}$ regarding the inability of such variable density generalizations of incompressible models to predict the strong influence of the convective Mach number on the growth rate of the shear layer. The new model of Sarkar et $a .^{6}$ for the compressible dissipation was then incorporated into a full Reynolds stress closure. The growth rates computed with this model, not only captured the experimentally observed sharp reduction of the growth rate at intermediate Mach numbers, but also showed the tendency to flatten out at large Mach numbers. The present calculations are also in agreement with the experimental result that the maximum normalized turbulence intensities and shear stress decrease when the convective Mach number is increased.

In the future, we propose to apply the present second-order closure to more complex compressible flows. Though, the consequences of the enhanced dissipation in compressible flows are consistent with some of the distinguishing features of the high-speed shear layer, 
other compressibility phenomena may become important in different flows like the shockboundary layer interaction. Our future studies will address issues relevant to the modeling of such distinct mechanisms.

\section{Acknowledgement}

The authors would like to thank Dr. Charles Speziale for his helpful comments regarding a preliminary draft of the manuscript. 


\section{References}

1. Papamoschou, D., and Roshko, A., "The Compressible Turbulent Shear layer: an Experimental Study," Journal of Fluid Mechanics, Vol. 197, 1988, pp. 453-477.

2. Elliott, G. S., and Samimy, M., "Compressibility Effects in Free Shear Layers," Physics of Fluids, Part A, 1990, to appear.

3. Oh, Y. H., "Analysis of Two-Dimensional Free Turbulent Mixing," AIAA Paper No. 74-594, June 1974.

4. Vandromme, D., "Contribution to the Modeling and Prediction of Variable Density Flows," Ph.D. Thesis, University of Science and Technology, Lille, France, 1983.

5. Dussauge, J. P., and Quine, C., "A Second-Order Closure for Supersonic Turbulent Flows: Application to the Supersonic Mixing," Workshop on the Physics of Compressible Turbulent Mixing, Princeton, Oct. 1988.

6. Sarkar, S., Erlebacher, G., Hussaini, M. Y., and Kreiss, H. O., "The Analysis and Modeling of Dilatational terms in Compressible Turbulence," ICASE Report 89-79, 1989.

7. Zeman, O., "Dilatational Dissipation: The Concept and Application in Modeling Compressible Mixing Layers," Physics of Fluids, Part A, 1990, to appear.

8. Lumley, J. L., "Computational Modeling of Turbulent Flows," Advances in Applied Mechanics, Vol. 18, 1978, pp. 123-176.

9. Lumley, J. L., "Turbulence Modeling," Journal of Applied Mechanics, Vol. 105, 1983, pp. 1097-1103. 
10. Launder, B. E., Reece, G. J., and Rodi, W., "Progress in the Development of a Reynolds-stress turbulence closure," Journal of Fluid Mechanics, Vol. 68, 1975, pp. $537-566$.

11. Shih, T-H., and Lumley, J. L., "Modeling of Pressure Correlation terms in Reynolds Stress and Scalar Flux Equations," Technical Report No. FDA-85-3, Cornell University, 1985.

12. Fu, S., Launder, B. E., and Tselepidakis, D. P., "Accommodating the Effects of High Strain Rates in Modeling the Pressure-Strain Correlation," UMIST Mechanical Engineering Department Report TFD/87/5, 1987.

13. Speziale, C. G., Sarkar, S., and Gatski, T. B., "Modeling the Pressure-Strain Correlation of Turbulence - An Invariant Dynamical Systems Approach," ICASE Report 90-5, 1990 .

14. Speziale, C. G., "Discussion of Turbulence Modeling: Past and Future," Lecture Notes in Physics, 1989, in press.

15. Erlebacher, G., Hussaini, M., Y., Kreiss, H. O., and Sarkar, S., "The Analysis and Simulation of Compressible Turbulence," ICASE Report 90-15, 1990.

16. Drummond, J. P., Rogers, R. C., and Hussaini, M. Y., "A Numerical Model for Supersonic Reacting Mixing Layers," Computer Methods in Applied Mechanics and Engineering, Vol. 64, 1987, pp. 39-60.

17. Carpenter, M.H., "The Effects of Finite Rate Chemical Processes on High Enthalpy Nozzle Performance: A Comparison between SPARK and SEAGULL," AIAA/ASME/SAE/ASEE 24 $4^{\text {th }}$ Joint Propulsion Conference, Boston, Massachusetts, July 1988. 
18. Kline, S. J., Cantwell, B. J., and Lilley, G. M., 1980-1981, AFOSR - HTTM Stanford Conference, Vol. 1, Stanford University Press, California, 1982, p. 368.

19. Wilcox, D. C., "Hypersonic Turbulence Modeling without the Epsilon Equation," Seventh National Aero-Space Plane Technology Symposium, Oct. 1989.

20. Free Turbulent Shear Flows, Vol. 1, July 1972, NASA SP-321.

21. Marvin, J. G., and Coakley, T. J., "Turbulence Modeling for Hypersonic Flows," 1989, NASA TM-101079.

22. Petrie, H. L., Samimy, M., and Addy, A. L., " A Study of Compressible Turbulent Free Shear Layers using Laser Doppler Velocimetry," AIAA Paper 85-0177, January 1985.

23. Ikawa, H., and Kubota, T., "Investigation of Supersonic Turbulent Mixing Layer with Zero Pressure Gradient," AIAA Journal, Vol. 13, No.5, 1975, pp. 566-572.

24. Wagner, R. D., "Mean Flow and Turbulence Measurements in a Mach 5 Free Shear Layer," NASA TN D-7966, December 1973. 


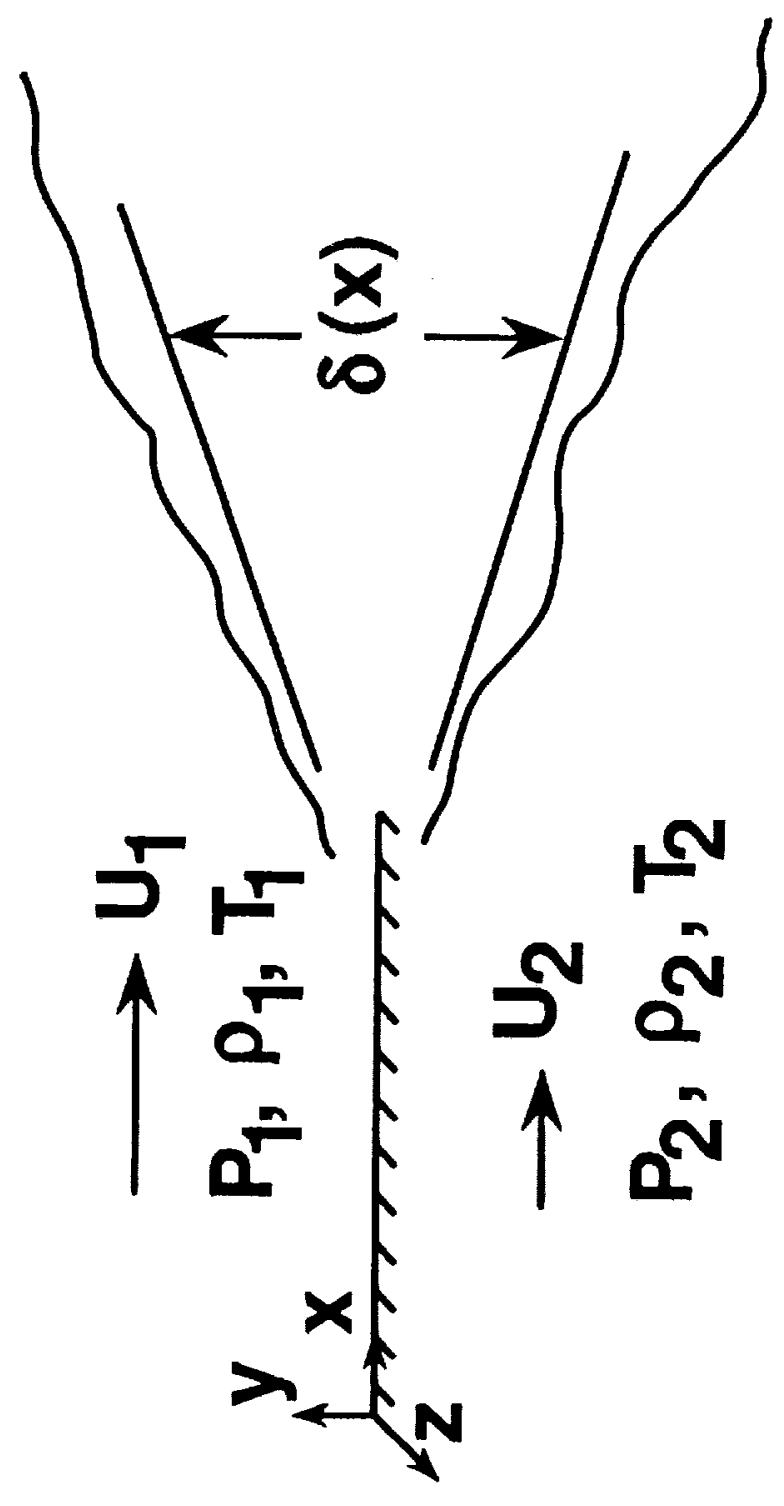

Figure 1. Schematic of the compressible shear layer. 


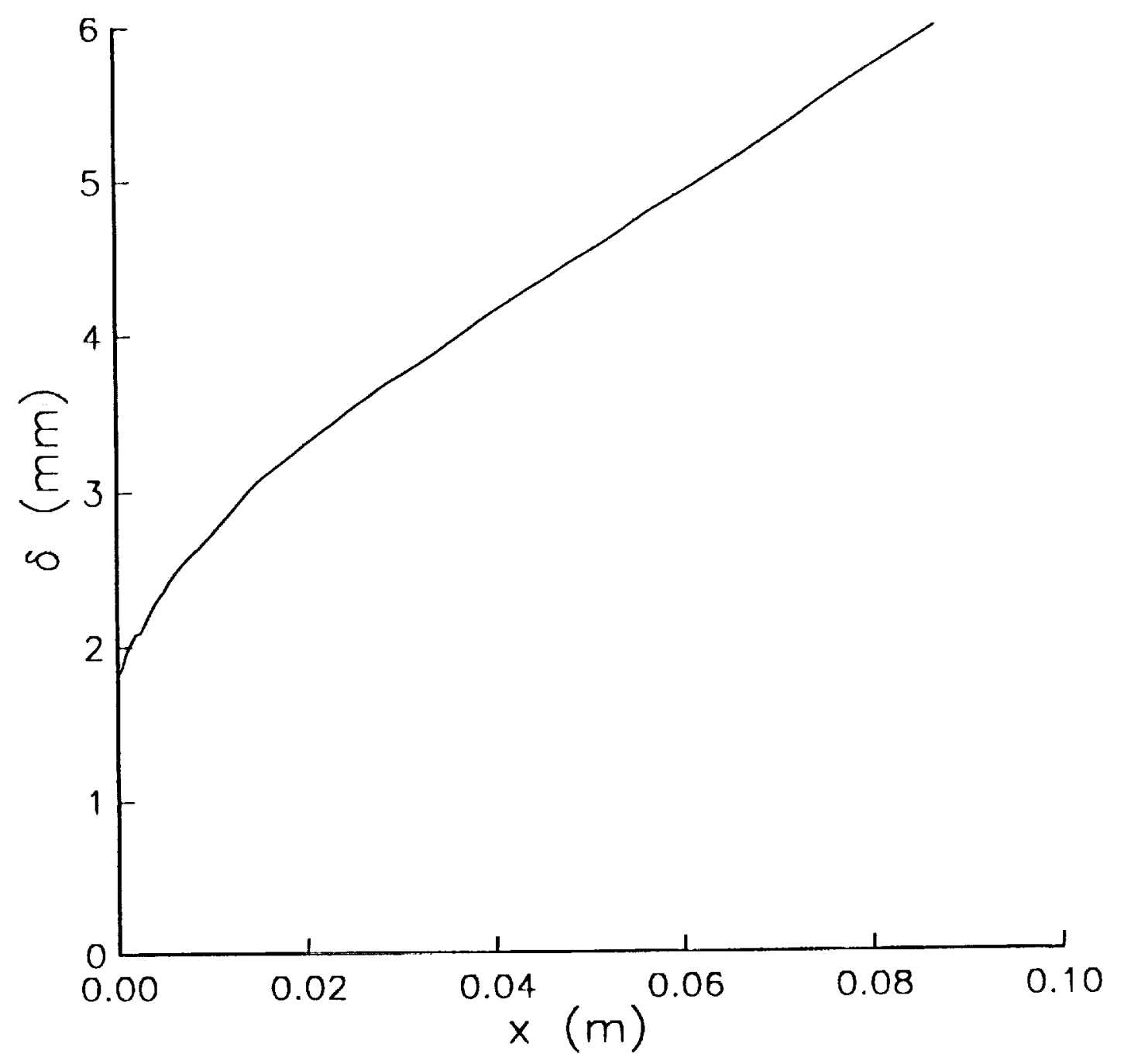

Figure 2. Downstream evolution of the shear layer thickness. 


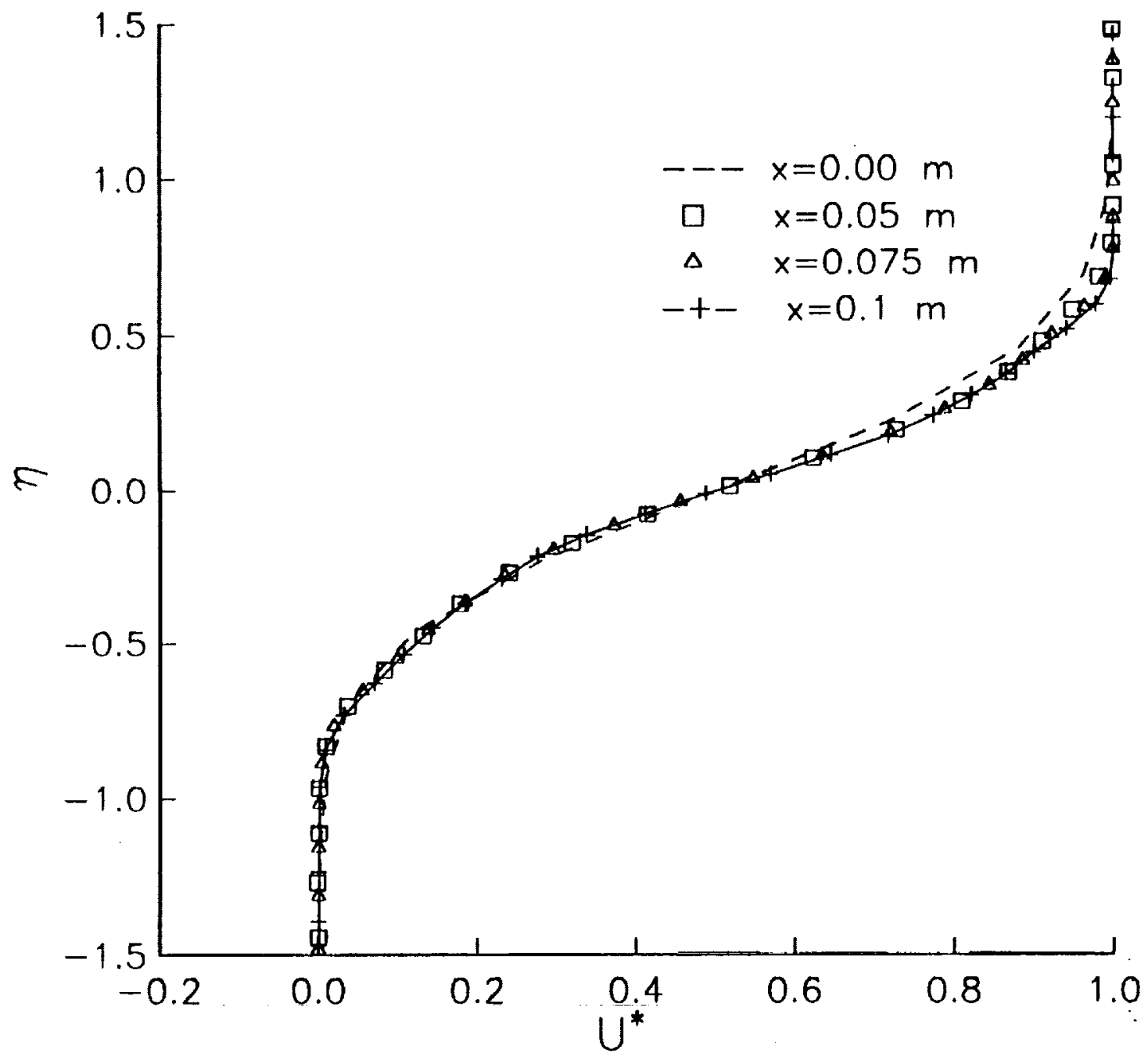

Figure 3. Transverse mean velocity profiles at various streamwise locations. 


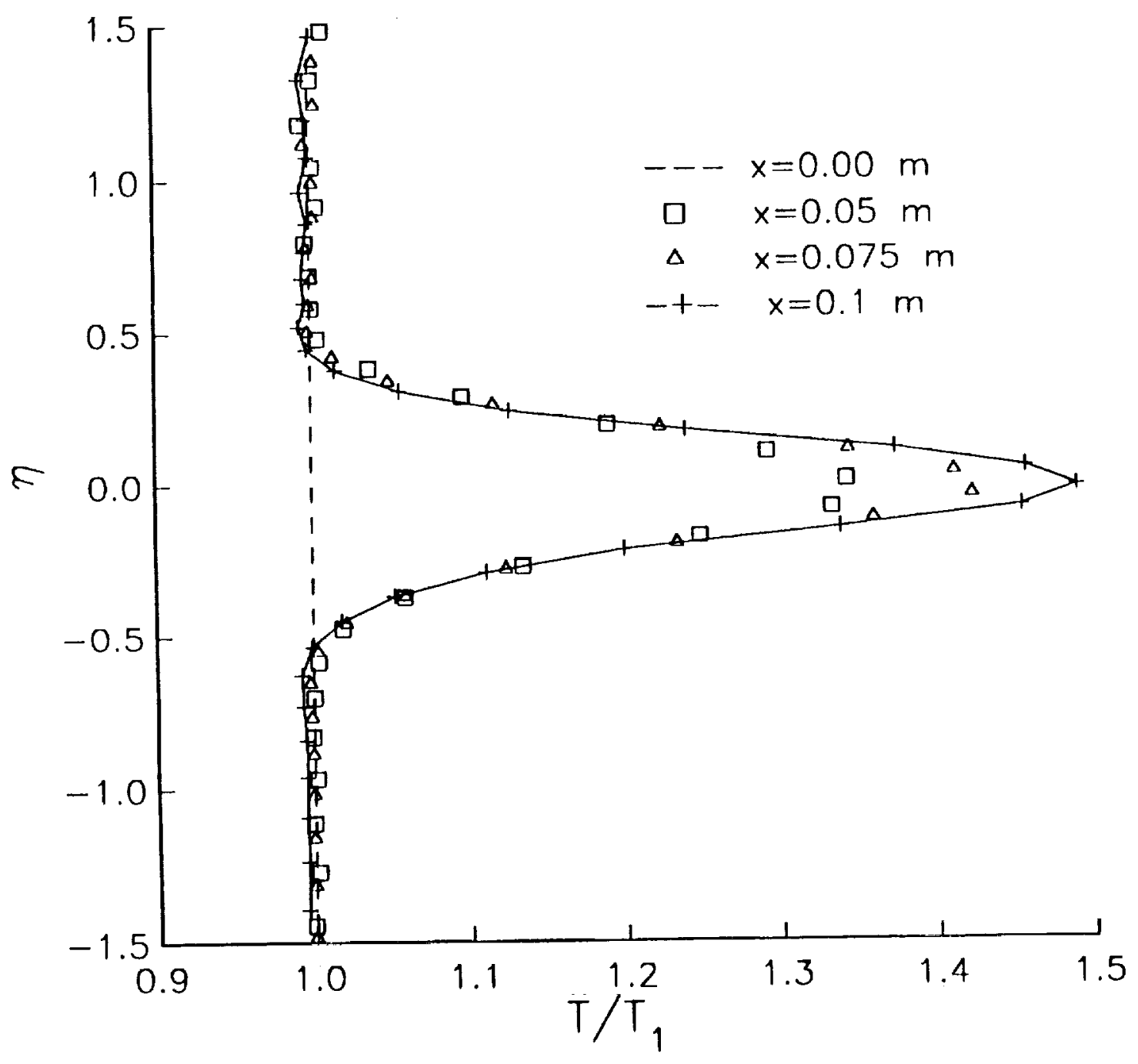

Figure 4. Transverse mean temperature profiles at various streamwise locations. 


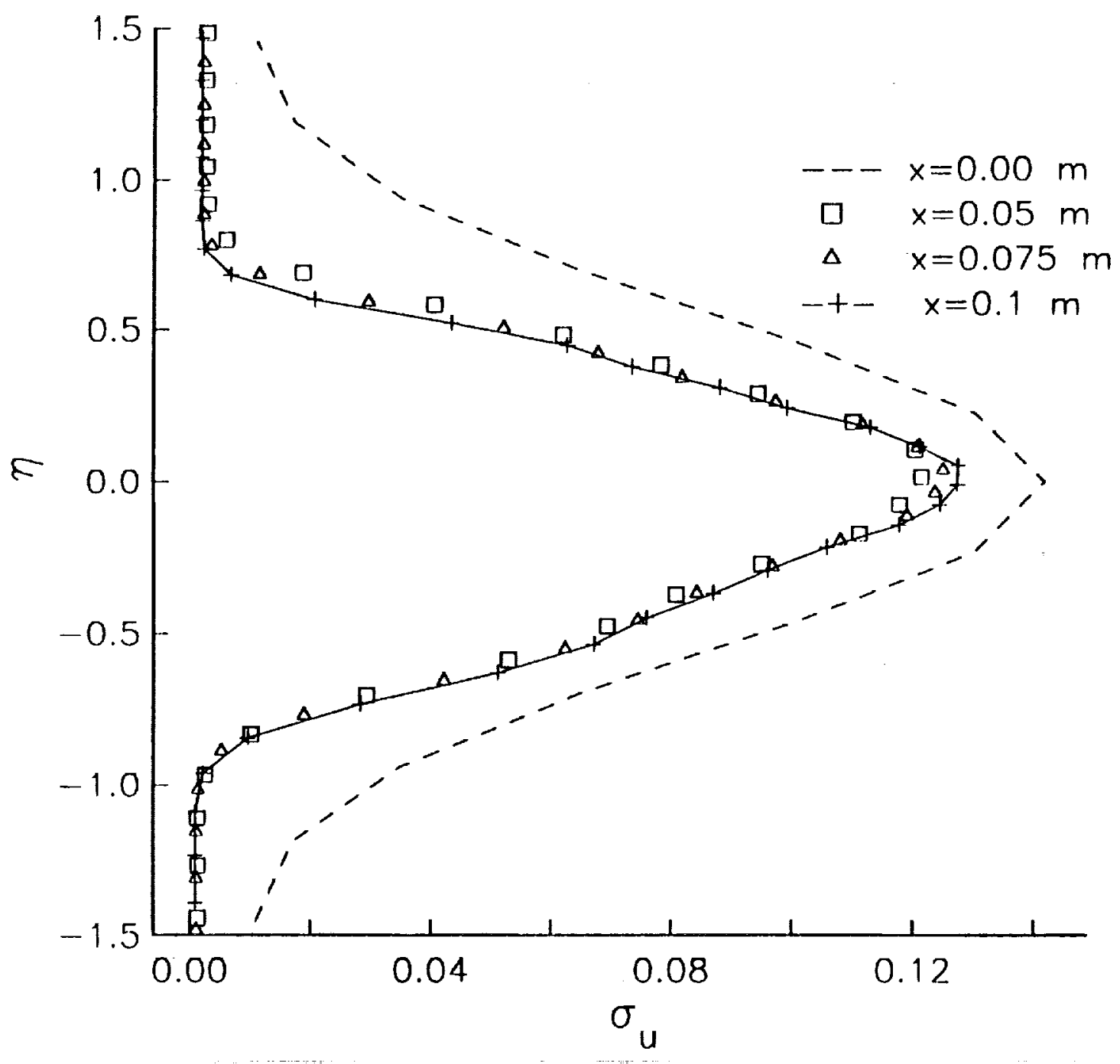

Figure 5. Transverse profiles of the streamwise component of the Reynolds stress tensor. 


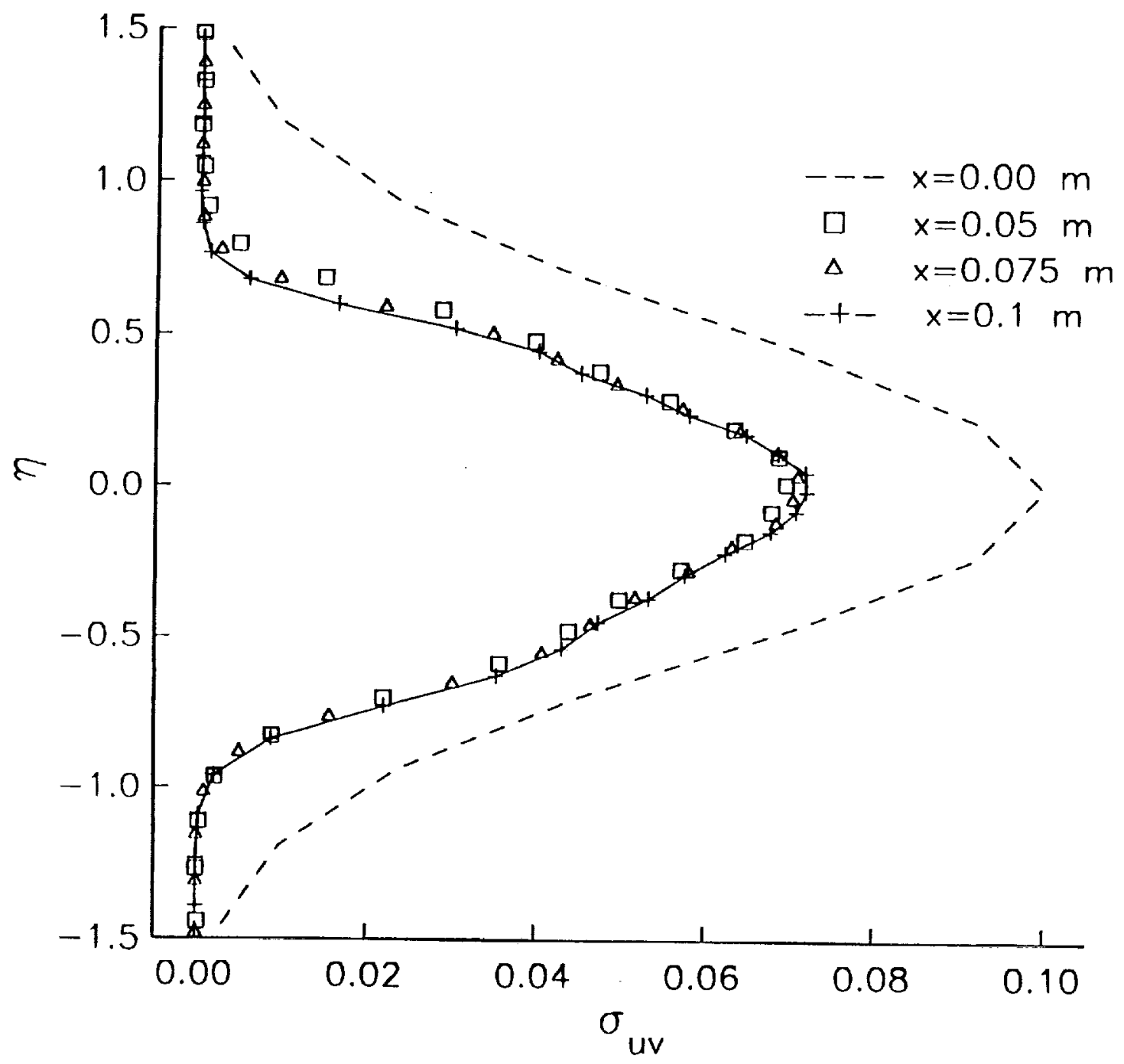

Figure 6. Transverse profiles of the Reynolds shear stress. 


\section{ORIGINAL PAGE IS OF. POOR QUALITY}

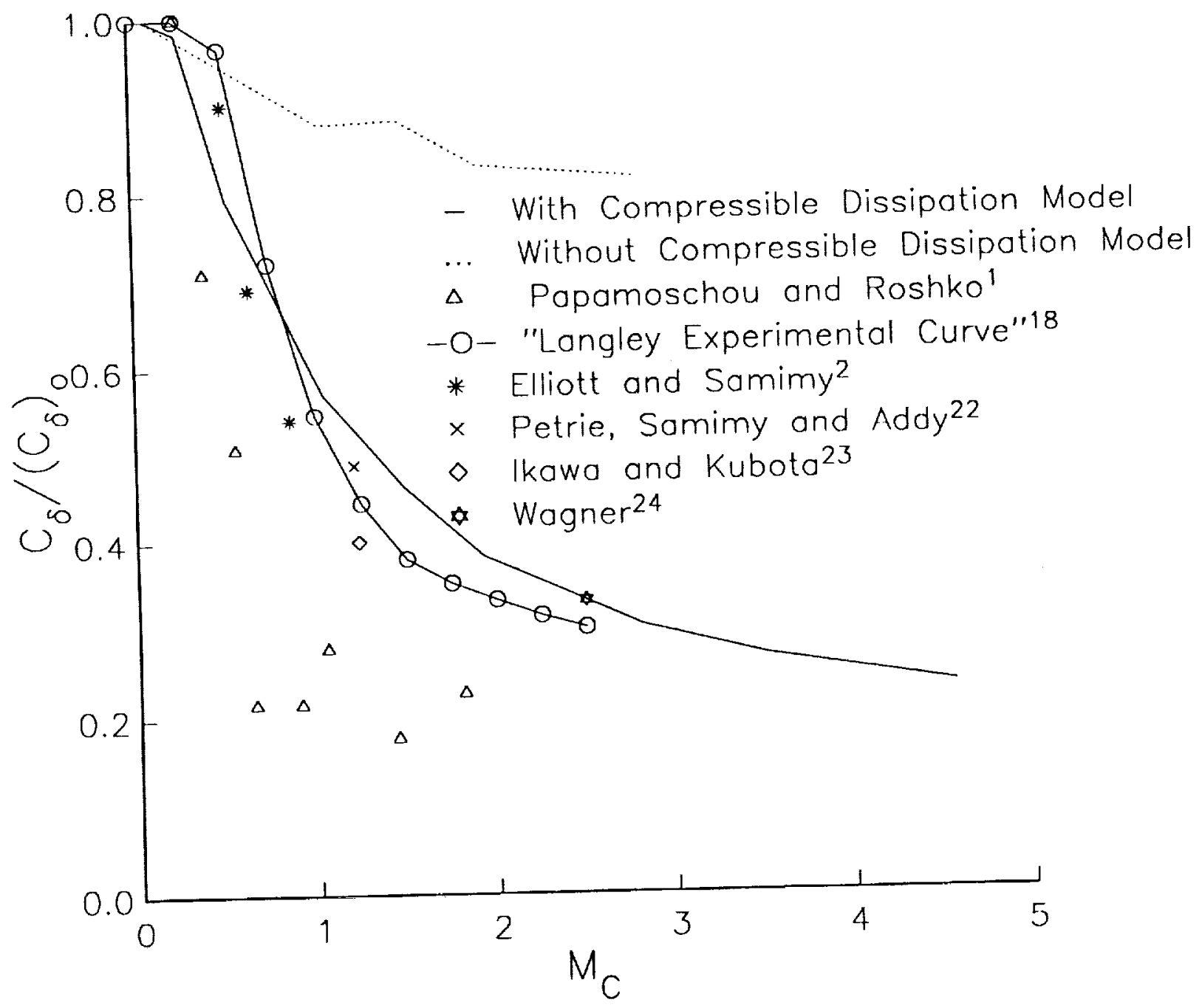

Figure 7. Variation of the growth rate of the compressible shear layer with the convective Mach number. 


\section{ORIGINAL PAGE IS \\ OF. POOR QUALITY}

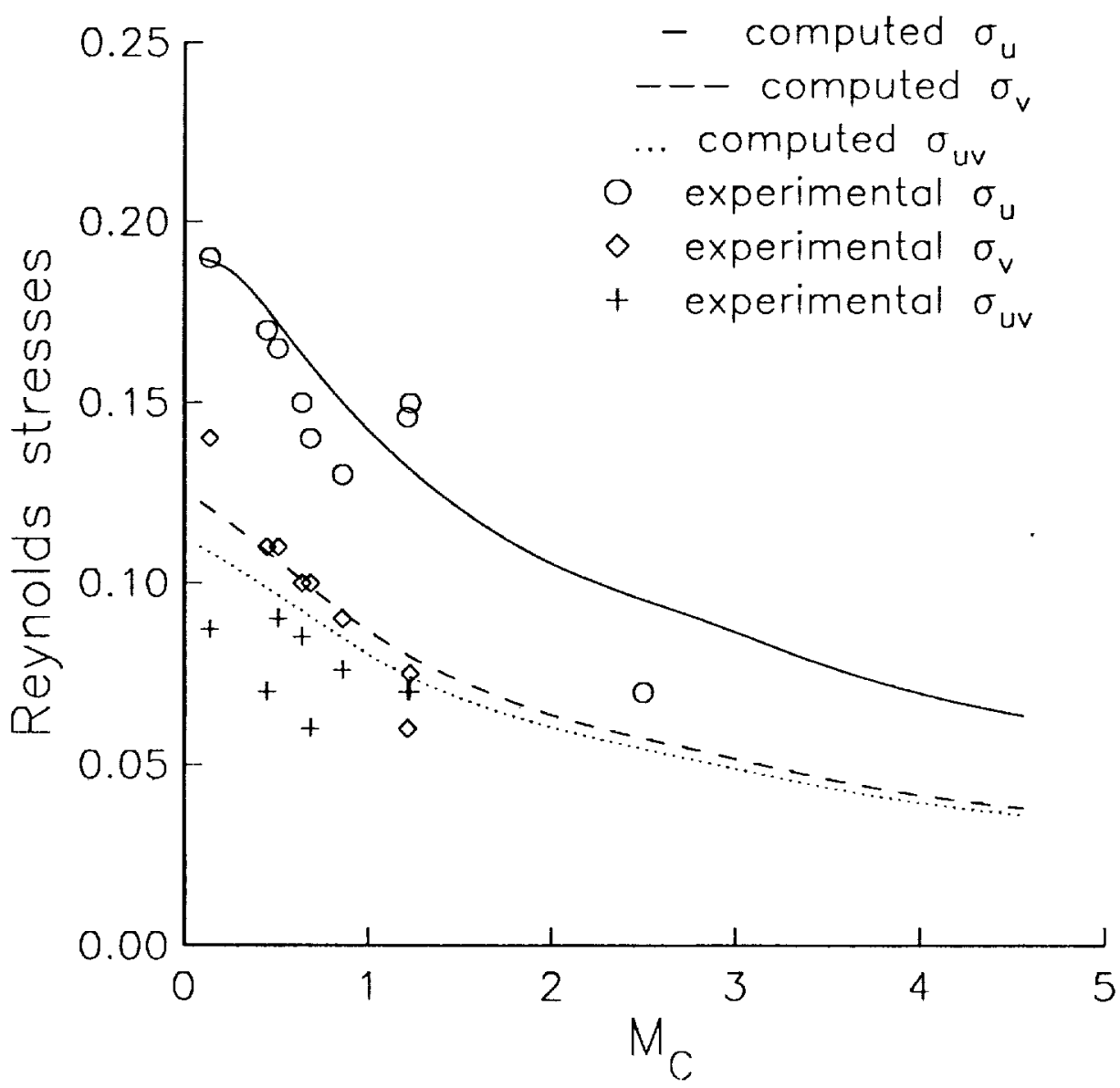

Figure 8. Variation of the maximum Reynolds stresses with the convective Mach number. 
and and

Wx $x-4$

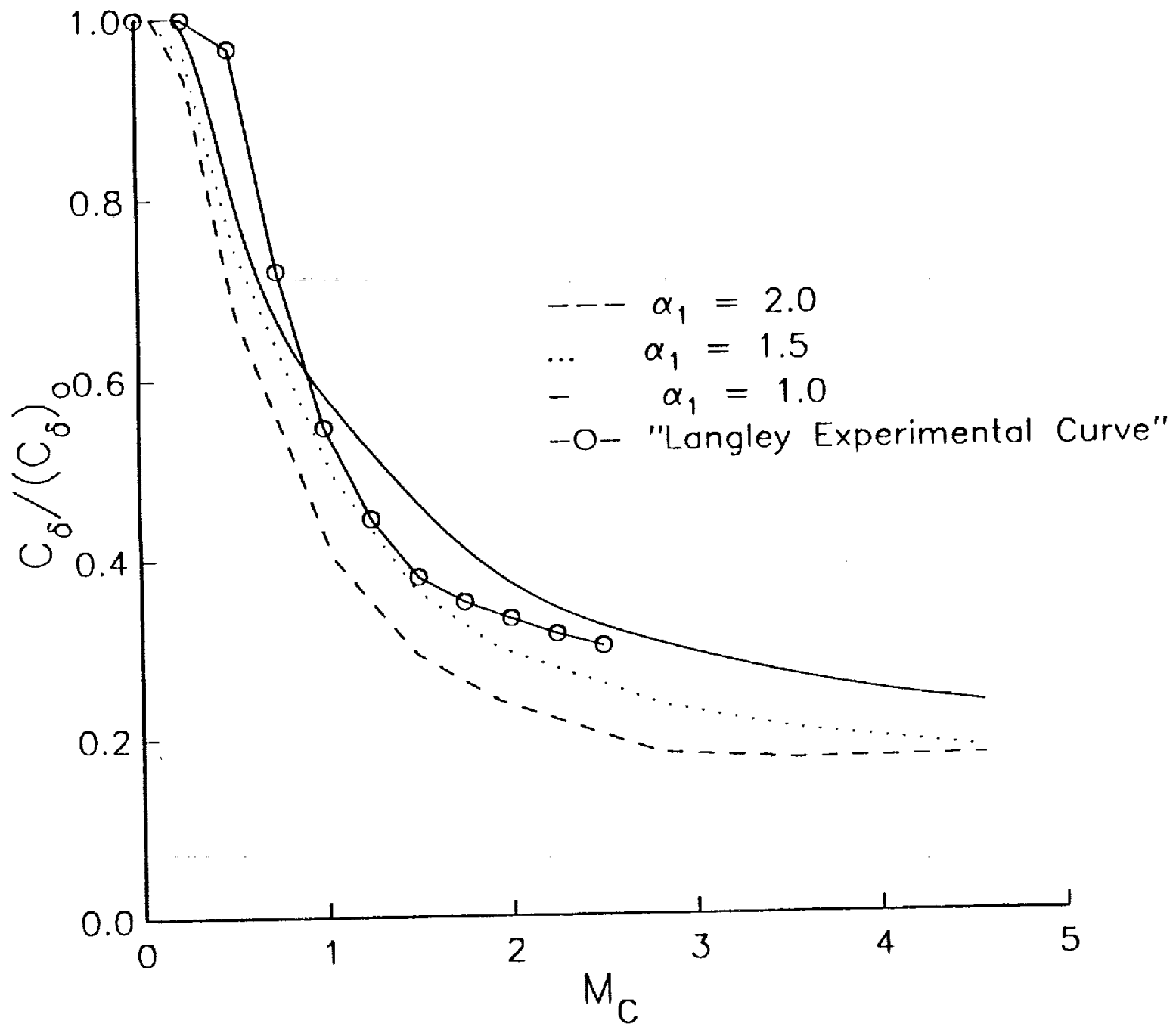

Figure 9. Computed growth rate curves fo: various values of the parameter $\alpha_{1}$ in the model for compressible dissipation. 


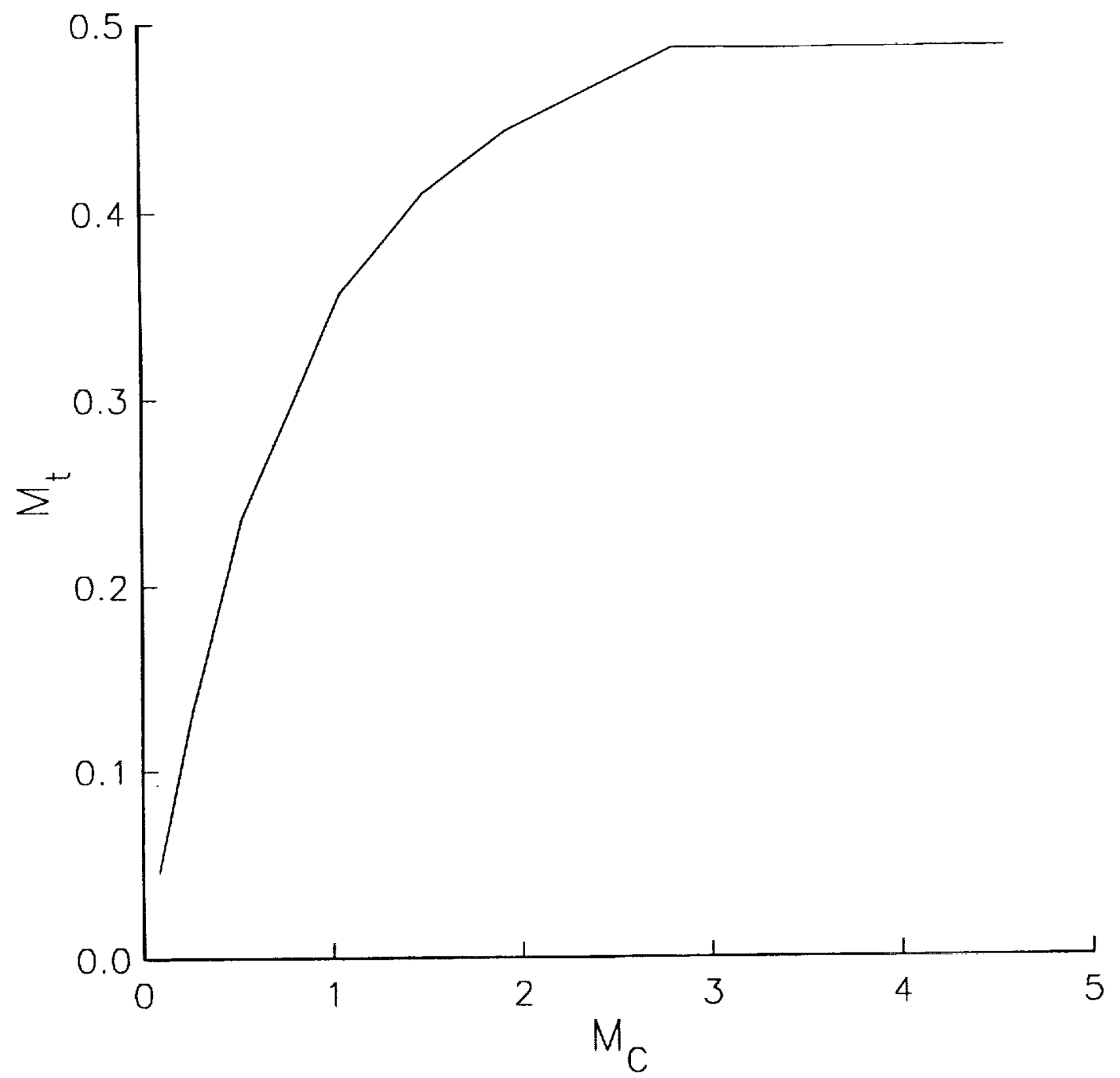

Figure 10. The dependence of the maximum computed value of the turbulent Mach number $M_{t}$ on the convective Mach number $M_{c}$. 


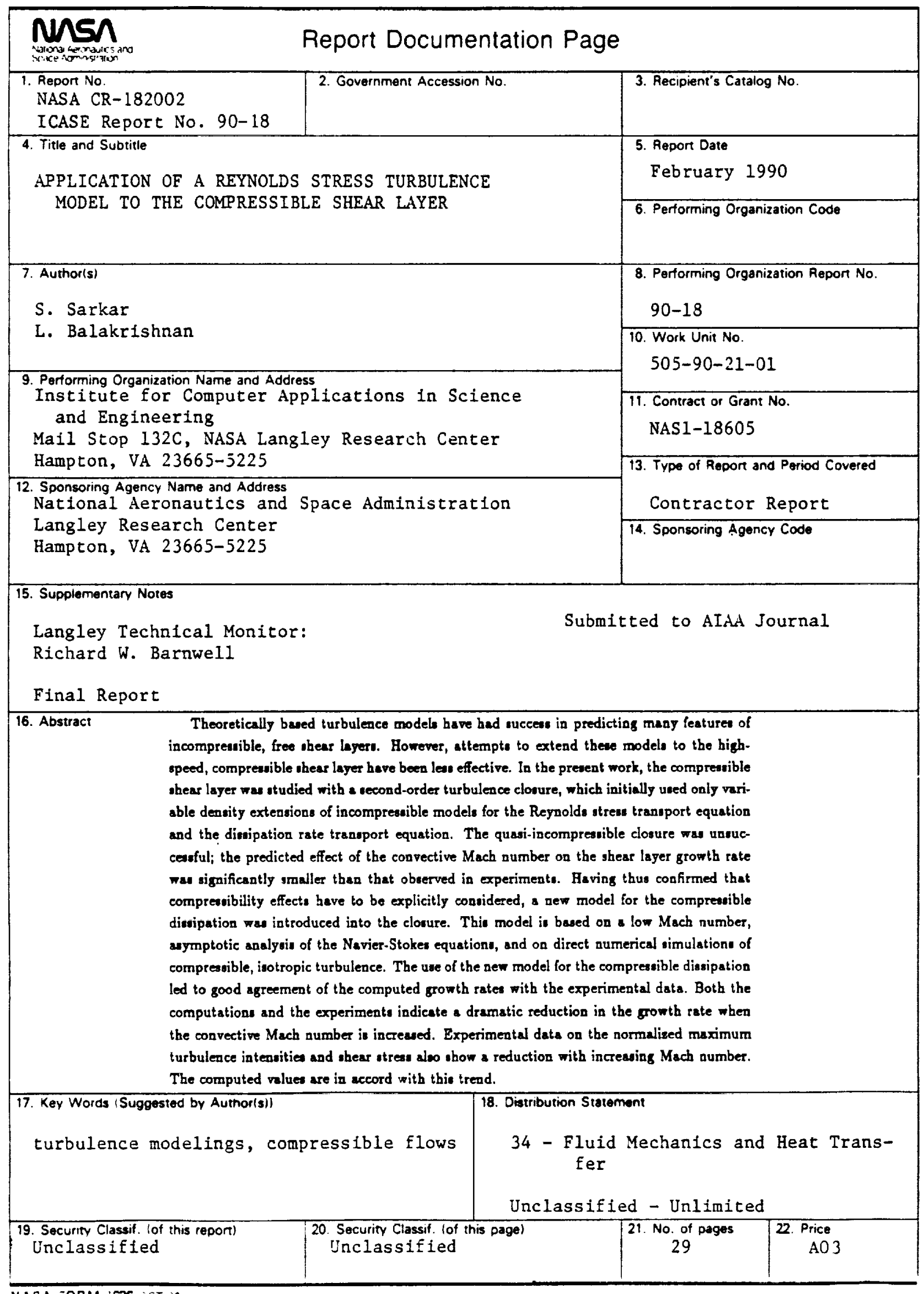

Vंडd :DRท $526: 0-: 6$ 
